\title{
PATENT DUCTUS ARTERIOSUS LIGATION IN PREMATURE INFANTS: UNPROVEN LONG TERM BENEFIT AT THE COST OF SHORT TERM MORBIDITIES?
}

\author{
B. Sinha ${ }^{1,2}$, B. Balahara ${ }^{3}$, A. Fujii ${ }^{1}$, S.A. Ringer ${ }^{2,4}$ \\ ${ }^{1}$ Pediatrics and Neonatology, Boston University School of Medicine, ${ }^{2}$ Harvard Medical School, ${ }^{3}$ Pediatrics, \\ Massachussetts General Hospital, ${ }^{4}$ Neonatology, Brigham and Women Hospital, Boston, MA, USA
}

Background and aims: Ligation goals for patent ductus arteriosus (PDA) in the preterm infant include prompt improvement in cardiorespiratory failure with rapid wean from mechanical ventilation and improvement in hemodynamic compromise leading to reduced inotropic support. However some infants who undergo PDA ligation do not demonstrate the expected dramatic improvement in cardiorespiratory status. The study was undertaken to asses whether PDA ligation causes short term deterioration in cardiopulmonary status as demonstrated by increase in ventilatory and vasopressor support in the post-operative period.

Design/methods: A retrospective chart review of 116 preterm infants $<32$ weeks gestation who underwent PDA ligation over a 5-year period was carried at a level 3 tertiary NICU . Demographics of babies with early extubation were compared with those with late extubation; babies with increased need for vasopressor (VP) support post ligation were compared with those not needing increased VP support. Data was analyzed using chi-square test, independent sample t-test and Wald test statistics for logistic regression.

Results: Approximately half of the babies (48.5\%) had the need for increased VP support post ligation. For 1 week reduction in gestational age, the odds of need for VP support post ligation and number of inotropes post ligation increased by $36.8 \%$ and $34.7 \%$ respectively while the odds of early extubation was two times less compared to late extubation.

Conclusions: PDA ligation causes short term deterioration in cardiopulmonary status in preterm babies. The cardiopulmonary deterioration was inversely related to gestational age and birth weight of the preterm infants. 